doi: $10.2306 /$ scienceasia1513-1874.2012.38.136

\title{
Oxidative stress status in patients with cardiac syndrome $\mathbf{X}$
}

\author{
Sam Seydi-Shirvani ${ }^{\mathrm{a}}$, Yousef Rasmi ${ }^{\mathrm{b}, \mathrm{c}, *}$, Mir-Hossein Seyyed-Mohammadzad ${ }^{\mathrm{d}}$, Fariba Khosravifar $^{\mathrm{a}}$ \\ a Department of Biochemistry, Payame - Noor University, P.O.Box 19395-3697, Tehran, Iran \\ b Centre for Cellular and Molecular Research, Urmia University of Medical Sciences, Urmia, Iran \\ c Department of Biochemistry, Faculty of Medicine, Urmia University of Medical Sciences, Urmia, Iran \\ d Department of Cardiology, Faculty of Medicine, Urmia University of Medical Sciences, Urmia, Iran
}

*Corresponding author, e-mail: rasmiy@umsu.ac.ir

Received 19 Oct 2011

Accepted 10 Apr 2012

\begin{abstract}
Cardiac syndrome X (CSX) is characterized by chest pain, typical angina pectoris, abnormal exercise test result and normal coronary arteries. Microvascular dysfunction and enhanced oxidative stress are the mechanisms suspected to play an important role in the pathogenesis of CSX. Thus we aimed to evaluate the oxidative stress status of 28 patients with CSX (14 male/14 female, mean age $49.5 \pm 9.3$ years) and 24 age- and gender-matched healthy controls (10 male/14 female, mean age $45.6 \pm 5.7$ years). Blood samples were drawn for measurement of malodialdehyde (MDA), as a marker of lipid peroxidation, glutathione (GSH) and superoxide dismutase (SOD) activity, as antioxidant markers and ferric reducing ability of plasma (FRAP), as a marker of total antioxidant capacity. There was significant increase in the levels of MDA in CSX patients comparing to controls ( $3.8 \pm 0.12$ vs $3.3 \pm 0.14 \mathrm{mM}$, respectively; $p=0.006)$. But the levels of FRAP in CSX patients were significantly lower than those controls ( $504 \pm 19$ vs $568 \pm 26 \mu \mathrm{M}$, respectively; $p=0.046)$. Also, GSH levels and SOD activity in patients were significantly lower than those of the controls (GSH: $133.6 \pm 5.4 \mathrm{vs} 152.5 \pm 7.8 \mathrm{mmol} / \mathrm{g}$ $\mathrm{Hb}, p=0.048$; SOD: $386 \pm 34$ vs $578 \pm 38 \mathrm{U} / \mathrm{g} \mathrm{Hb}, p=0.0001$ ). It may be concluded that there is systemic oxidative stress in CSX patients. Considerable changes of antioxidant concentrations, indicating a compensatory mechanism to cope with increased oxidative stress in CSX patients and the body's antioxidant defence mechanisms try to minimize oxidative stress damage.
\end{abstract}

KEYWORDS: microvascular dysfunction, antioxidant, angina pectoris

\section{INTRODUCTION}

Cardiac syndrome $\mathrm{X}(\mathrm{CSX})$ is defined by no significant coronary stenosis in patients with typical angina pectoris and abnormal exercise test results ${ }^{1}$, and $1-12 \%$ of individuals with myocardial infarction who undergo coronary angiography are found to have normal coronary arteries ${ }^{2}$. The aetiology and pathophysiology of CSX is complex. Microvascular abnormalities, caused by coronary endothelial dysfunction, appear to contribute to the myocardial ischaemia in patients with $\mathrm{CSX}^{3}$, linked to risk factors such as hypertension, hypercholesterolaemia, diabetes mellitus, and smoking where high levels of oxidative stress are known to exist ${ }^{4,5}$.

On the other hand, growing evidence suggests that high reactive oxygen species (ROS) of endogenous or environmental origin play a key role in the genesis and progression of various vascular diseases 6,7 . Malodialdehyde (MDA) is a decomposition product of autooxidation of polyunsaturated fatty acids commonly used as an index of oxidative damage ${ }^{8,9}$. High MDA concentrations indicate increased membrane lipid peroxidation. The rise in MDA could be due to increased generation of ROS due to excessive oxidative damage. These oxygen species in turn, can oxidize many other important bimolecules including membrane lipids ${ }^{8}$. Normally these ROS are effectively kept under control by the various levels of antioxidant defences. Imbalance of this reaction either due to excess free radical formation or insufficient removal by antioxidants leads to oxidative stress ${ }^{7}$. The biological effects of ROS are controlled by many non enzymatic antioxidant such as glutathione (GSH) and vitamin $\mathrm{E}$ as well as enzymes such as catalase, superoxide dismutase (SOD) and various peroxides ${ }^{10}$, that have the ability to inhibit oxidative stress by scavenging the highly destructive free radicals ${ }^{11}$. The present study was designed to investigate the extent of oxidative stress in patients with CSX. 


\section{MATERIALS AND METHODS}

\section{Study population}

Between October 2009 and January 2010, 28 consecutive patients (14 male/14 female, mean age: $49.5 \pm 9.3$ years) who underwent angiography in the Department of Cardiology at Urmia University of Medical Sciences, Urmia, Iran; and diagnosed with CSX were considered for inclusion into our study. Entry criteria were typical anginal chest pain, a positive exercise ECG stress test response, and a normal coronary angiogram. The control group consisted of 24 apparently healthy subjects (14 females/10 males; mean age: $45.6 \pm 5.7$ years). None of the control subjects had a previous history of chest pain or acute/chronic diseases. The study was conducted in accordance with the Declaration of Helsinki and its revisions and was approved by the research ethics committee of our university. All subjects gave written informed consent. After demographics were recorded and informed consent obtained, their medication history, smoking habit, and other relevant data were provided from related questionnaires.

\section{Exclusion criteria}

Noncardiac causes of chest pain, such as gastrointestinal and musculoskeletal disorders, were also investigated and ruled out as appropriate. Patients with diabetes mellitus were not included either, due to confirmed changes in oxidative stress status. In addition, none of the controls were taking medications.

\section{Blood collection}

An 8-ml heparinzed blood sample was obtained from each subject. Then $2 \mathrm{ml}$ of whole blood was transferred into a labelled vial for evaluation of SOD activity and GSH concentration and $6 \mathrm{ml}$ of blood was centrifuged at $2000 \mathrm{~g}$ for $15 \mathrm{~min}$. Plasma was aliquoted and stored at $-80^{\circ} \mathrm{C}$ until analysis. Samples were analysed for haemoglobin $(\mathrm{Hb}), \mathrm{MDA}$, ferric reducing antioxidant power or ferric reducing ability of plasma (FRAP), GSH, and SOD activity. Hb levels were measured using a Sysmex automated haematology analyser.

\section{Estimation of malondialdehyde}

Measurement of MDA were estimated in plasma by a standard technique ${ }^{12}$. Briefly, the pink chromogen produced by the reaction of thiobarbituric acid with malondialdehyde, a secondary product of lipid peroxidation was estimated. The absorbance of clear supernatant was measured against reference blank at $535 \mathrm{~nm}$. Results were expressed as $\mathrm{mM}$ for plasma.
Table 1 Demographics and characteristics of CSX patients and controls.

\begin{tabular}{lccc}
\hline & CSX & Control & $p$ value \\
\hline Gender (male/female) & $14 / 14$ & $10 / 14$ & 0.822 \\
Age (years) & $49.5 \pm 9.3$ & $45.6 \pm 5.7$ & 0.770 \\
Smokers (yes/no) & $11 / 17$ & $7 / 17$ & 0.780 \\
BMI $\left(\mathrm{kg} / \mathrm{m}^{2}\right)$ & $27.0 \pm 5.3$ & $25.7 \pm 3.0$ & 0.297 \\
History of hyper- & $12(42.8)$ & $0(0)$ & $<0.0001$ \\
tension $(n(\%))$ & & & \\
\hline
\end{tabular}

\section{Determination of SOD activity}

SOD activity was assayed by spectrophotometer in whole blood with "Ransod" kit (Randox Laboratories Ltd, UK).

\section{Estimation of glutathione}

Glutathione was determined in whole blood based on Butler's method ${ }^{13}$. Briefly, 5,5'-dithiobis-(2-nitrobenzoic acid) (DTNB) is a disulphide chromogen that is readily reduced by sulphydryl compounds to an intensely yellow compound. The absorbance of the reduced chromogen is measured at $412 \mathrm{~nm}$.

\section{Estimation of total antioxidant capacity}

Total antioxidant capacity was determined by FRAP method $^{14}$. Briefly, $300 \mathrm{mM}$ of acetate buffer ( $\left.\mathrm{pH} 3.6\right)$, $10 \mathrm{mmol} / \mathrm{l}$ 2,4,6-tri-pyridyl-s-triazine (TPTZ) in $40 \mathrm{mM} \mathrm{HCl}$ and $20 \mathrm{mM} \mathrm{FeCl}_{3}$ in the ratio of 10:1:1 give the working FRAP reagent. Then, $750 \mu \mathrm{l}$ of working FRAP reagent was mixed with $25 \mu \mathrm{l}$ plasma or standard in a test tube. After exactly $10 \mathrm{~min}$ at room temperature, the absorbance at $593 \mathrm{~nm}$ was read against reagent blank. Fe(II) standards are used. $\mathrm{Fe}(\mathrm{II})(1000 \mu \mathrm{M})$ is equivalent to $1000 \mu \mathrm{M}$ of FRAP. The change in absorbance is directly related to the "total" reducing power of the electron-donating antioxidants present in the reaction mixture.

\section{Statistical analysis}

For statistical analysis, chi-square and student $t$ tests were carried out using SPSS software (version 16.0). $P$ values less than 0.05 were considered statistically significant. Results are expressed as mean $\pm \mathrm{SD}$.

\section{RESULTS}

Table 1 shows the age, gender, smoking habits, and BMI in CSX patients and controls. No statistically significant difference was observed between patients and controls for age, gender, smoking habits, and BMI $(p>0.05)$. 
Table 2 Oxidative stress status in CSX patients and controls.

\begin{tabular}{lccl}
\hline & CSX & Control & $p$ value \\
\hline MDA $(\mathrm{mM})$ & $3.8 \pm 0.12$ & $3.3 \pm 0.14$ & 0.006 \\
FRAP $(\mu \mathrm{M})$ & $504 \pm 19$ & $568 \pm 26$ & 0.046 \\
$\mathrm{GSH}(\mathrm{mmol} / \mathrm{g} \mathrm{Hb})$ & $133.6 \pm 5.4$ & $152.5 \pm 7.8$ & 0.048 \\
$\mathrm{SOD}(\mathrm{U} / \mathrm{g} \mathrm{Hb})$ & $386 \pm 34$ & $578 \pm 38$ & 0.0001 \\
$\mathrm{Hb}(\mathrm{g} / \mathrm{dl})$ & $13.3 \pm 0.4$ & $14.1 \pm 0.3$ & 0.093 \\
\hline
\end{tabular}

In CSX patients, the mean plasma MDA levels was significantly higher than in controls $(p=0.006)$, whereas, the mean plasma FRAP and GSH levels in CSX were significantly lower than those in controls ( $p=0.046$ and 0.048 , respectively). Also, the mean SOD activity in CSX was significantly lower than those of the control group ( $p=0.0001)$. No statistically significant difference was observed between patients and controls for $\mathrm{Hb}(p=0.093)$, Table 2 .

\section{DISCUSSION}

The classical definition of CSX is characterized by angina-like pain on effort, ST-segment depression on exercise stress test and angiographically normal coronary arteries, in the absence of any other cardiac diseases and of systemic diseases known to influence vascular function ${ }^{15,16}$. Myocardial ischaemia because of coronary microvascular dysfunction has been guessed to be responsible for $\mathrm{CSX}^{17,18}$. Recently, we hypothesized that CSX pathophysiology emphasizes a dysfunctional vascular endothelium that leads to microvascular ischaemia ${ }^{19}$.

Microvascular dysfunction was reported to be associated with proinflammatory cytokines or oxidative stress $^{20,21}$. Oxidative stress is thought to be linked to certain cardiovascular diseases. In the literature, there are only two studies revealing the association between CSX and oxidative stress that have shown decreased total antioxidant levels in CSX patients ${ }^{22,23}$.

In this study, lipid peroxidation was evaluated by measuring of MDA, which results from free radical damage to the membrane components of the cells and can also deactivate membrane transporters, by forming intra- and inter-molecular crosslinks ${ }^{24,25}$. We found a significant increase in the MDA concentration in CSX patients. Increased MDA is thought to be a result of oxidative stress, which occurs when the balance between prooxidant and antioxidant mechanism is impaired.

Also, to clarify the contribution of oxidative stress to the association of the CSX disease, we evaluated the SOD activity in CSX patients and controls because
SOD has been reported to be the major antioxidant enzyme system of the arterial vessel wall ${ }^{26}$. In this study, SOD activity was significantly lower CSX patients than in controls individuals. SOD plays a very important role in the protection against lipid peroxidation. The decrease in the activity of SOD could be due to inactivation of the enzymes by crosslinking or due to exhaustion of the SOD by increased peroxidation

Also, for the first time - to our knowledge-in the literature, we observed a significant decrease in the levels of erythrocyte GSH in CSX patients, when compared to controls. The decrease in the levels of GSH may be due to an increased turnover, for preventing oxidative damage in CSX patients, suggesting an increased defence against oxidant damage in coronary microvascular dysfunction. The deleterious influences of free radicals are kept under check by a delicate balance between the rate of their production and the rate of their elimination by these defence systems ${ }^{8,27}$. Likewise, we have shown reduced FRAP levels in CSX patients.

Considerable changes of FRAP indicate a compensatory mechanism(s) to cope up with increased oxidant parameters in CSX patients. However, the body's antioxidant defence mechanisms try to minimize this damage.

Our finding of increased oxidative stress markers such as MDA and decreased levels of some important antioxidant such as FRAP (total antioxidant), GSH, and SOD activity in patient with CSX compared to healthy controls indicate that increased oxidative stress that has an association in pathogenesis of CSX. These findings indicate that increased MDA can occur if the rate of production of antioxidant substances is low. Low level of antioxidants, or inhibition of the antioxidant enzymes, cause oxidative stress and may damage or kill cells ${ }^{28}$.

Thus in the present study, the increase in oxidative stress markers such as MDA levels and the decrease in antioxidant markers such as FRAP and GSH and SOD activity correlated with the presence of CSX, supports the reports regarding the role of oxidative stress in the pathogenesis of CSX ${ }^{22,23,29}$. However, the role of oxidative stress in patient with CSX has not been fully elucidated and we could not find many data in literature about the oxidative stress statusparticularly stated parameters-in CSX patients.

\section{CONCLUSIONS}

In conclusion, with findings of the present study, we have demonstrated a significant decrease in FRAP and GSH levels and SOD activity and increase in MDA 
levels in patients with CSX, indicating increased oxidative stress in CSX that might play a role in the pathogenesis of microvascular dysfunction in patients with CSX. Our results indicate that patients with CSX are under the influence of oxidative stress because of lower levels of antioxidants markers. Decreasing oxidative stress, with antioxidant medications or supplementations, may be a novel treatment strategy in cases with CSX in the future ${ }^{23}$. Elevated antioxidants levels after use of medications or supplementations, indicator of decreased oxidative stress, might be the potential therapeutic target for treating cardiovascular diseases related with oxidative stress. Further studies are needed to clarify the mechanism(s) leading to these results.

Acknowledgements: This work derived from a Master of Science thesis in biochemistry and supported by research grant from the Urmia University of Medical Sciences, Urmia, Iran.

\section{REFERENCES}

1. Kaski JC (2006) Cardiac syndrome X in women: the role of oestrogen deficiency. Heart 92, Suppl 3:iii 5-9.

2. Ammann P, Marschall S, Kraus M, Schmid L, Angehrn W, Krapf R, Rickli H (2000) Characteristics and prognosis of myocardial infarction in patients with normal coronary arteries. Chest 117, 333-8.

3. Hurst T, Olson TH, Olson LE, Appleton CP (2006) Cardiac syndrome $\mathrm{X}$ and endothelial dysfunction: new concepts in prognosis and treatment. Am J Med 119, 560-6.

4. Kaski JC, Aldama G, Cosín-Sales J (2004) Cardiac syndrome X. Diagnosis, pathogenesis and management. Am J Cardiovasc Drugs 4, 179-94.

5. Elhadd TA, Khan F, Kirk G, McLaren M, Newton RW, Greene SA, Belch JJ (1998) Influence of puberty on endothelial dysfunction and oxidative stress in young patients with type 1 diabetes. Diabetes Care 21, 1990-6.

6. Marx JL (1987) Oxygen free radicals linked to many diseases. Science 235, 529-31.

7. Risal S, Adhikari D, Alurkar VM, Singh PP (2006) Oxidative stress and antioxidant status in cardiovascular diseases in population of western Nepal. Kathmandu Univ Med J 4, 271-4.

8. Aparna P, Betigeri A, Pasupathi P (2010) Homocysteine and oxidative stress markers and inflammation in patients with coronary artery disease. Int J Biol Med Res 1, 125-9.

9. Cavalca V, Cighetti G, Bamonti F, Loaldi A, Bortone L, Novembrino C, De Franceschi M, Belardinelli R, Guazzi MD (2001) Oxidative stress and homocysteine in coronary artery disease. Clin Chem 47, 887-92.

10. Dikici I, Mehmetoglu I, Dikici N, Bitirgen M, Kurban S (2005) Investigation of oxidative stress and some antioxidants in patients with acute and chronic viral hepatitis B and the effect of interferon-alpha treatment. Clin Biochem 38, 1141-4.

11. Halliwell B (1994) Free radicals and antioxidants: a personal view. Nutr Rev 52, 253-65.

12. Kamal A, Gomaa A, El Khafif M, Hammad A (1989) Plasma lipid peroxides among workers exposed to silica or asbestos dusts. Environ Res 49, 173-80.

13. Butler RN, Butler WJ, Moraby Z, Fettman MJ, Khoo KK, Roberts-Thomson IC (1994) Glutathione concentrations and glutathione S-transferase activity in human colonic neoplasms. J Gastroenterol Hepatol 9, 60-3.

14. Benzie IFF, Strain JJ (1996) The ferric reducing ability of plasma (FRAP) as a measure of "antioxidant power": the FRAP assay. Anal Biochem 239, 70-6.

15. Crea F, Lanza GA (2004) Angina pectoris and normal coronary arteries: cardiac syndrome X. Heart 90, 457-63.

16. Kemp HG Jr (1973) Left ventricular function in patients with the anginal syndrome and normal coronary arteriograms. Am J Cardiol 32, 375-6.

17. Chauhan A, Mullins PA, Taylor G, Petch MC, Schofield PM (1997) Both endothelium-dependent and endothelium-independent function is impaired in patients with angina pectoris and normal coronary angiograms. Eur Heart J 18, 60-8.

18. Buffon A, Rigattieri S, Santini SA, Ramazzotti V, Crea F, Giardina B, Maseri A (2000) Myocardial ischemiareperfusion damage after pacing-induced tachycardia in patients with cardiac syndrome X. Am J Physiol 279, H2627-33.

19. Rasmi Y, Raeisi S (2009) Possible role of Helicobacter pylori infection via microvascular dysfunction in cardiac syndrome X. Cardiol J 16, 585-7.

20. Vane JR, Anggard EE, Botting RM (1990) Regulatory functions of the vascular endothelium. New Engl J Med 323, 27-36.

21. Osamichi S, Kouji K, Yoshimaro I, Tadashi U, Hiroichi T, Seiyu K, Shinji O, Noboru T (2004) Myocardial glucose metabolism assessed by positron emission tomography and the histopathologic findings of microvessels in syndrome X. Circ J 68, 220-6.

22. On YK, Park R, Hyon MS, Kim SK, Kwon YJ (2005) Are low total serum antioxidant status and elevated levels of C-reactive protein and monocyte chemotactic protein-1 associated with cardiac syndrome X? Circ J 69, 1212-7.

23. Gur M, Yildiz A, Demirbag R, Yilmaz R, Aslan M, Ozdogru I, Erel O (2007) Paraoxonase and arylesterase activities in patients with cardiac syndrome $\mathrm{X}$, and their relationship with oxidative stress markers. Coron Artery Dis 18, 89-95.

24. Kramer JH, Mak IT, Weglicki WB (1984) Differential sensitivity of canine cardiac sarcolemmal and microsomal enzymes to inhibition by free radical-induced lipid peroxidation. Circ Res 55, 120-4.

25. Rajguru SU, Yeargans GS, Seidler NW (1994) Exercise 
causes oxidative damage to rat skeletal muscle microsomes while increasing cellular sulfydryls. Life Sci $\mathbf{5 4}$, 149-57.

26. Celik T, Iyisoy A, Kursaklioglu H, Kardesoglu E, Kilic S, Turhan H, Yilmaz MI, Ozcan O, Yaman H, Isik E, et al (2006) Comparative effects of nebivolol and metoprolol on oxidative stress, insulin resistance, plasma adiponectin and soluble P-selectin levels in hypertensive patients. J Hypertens 24, 591-6.

27. Shrinivas K, Bhaskar M, Aruna Kumari R, Nagaraj K, Reddy K (2000) Antioxidants, lipid peroxidation and lipoproteins in primary hypertension. Indian Heart $J$ 52, 285-8.

28. Aviram M (2000) Review of human studies on oxidative damage and antioxidant protection related to cardiovascular diseases. Free Radic Res 33, Suppl: S85-97.

29. Erdamar H, Sen N, Tavil Y, Yazici HU, Turfan M, Poyraz F, Topal S, Okuyan H, Cemri M, Cengel A (2009) The effect of nebivolol treatment on oxidative stress and antioxidant status in patients with cardiac syndrome-X. Coron Artery Dis 20, 238-4. 